\title{
Les agressions à Cologne au prisme de doxaï et d'enjeux politiques : construction et naturalisation des identités
}

\author{
Véronique Magaud \\ Université Catholique de Lyon
}

\section{Introduction}

La parole politique, dans le cadre de l'accueil des réfugiés, est plus révélatrice des tensions politiques qui traversent la société française que de réflexions sur les dysfonctionnements politiques aux niveaux national et international. Les agressions à Cologne constituent l'acmé de ces récupérations politiciennes et médiatiques qui accommodent ces événements aux enjeux et luttes politiques du moment. C'est donc autour de cet événement médiatisé que nous souhaitons interroger la construction et la nature des identités autour du Soi et de l'Autre à travers les prises de position de l'extrême-droite et de la droite en France, respectivement, Marine Le Pen (Front National) et Valérie Boyer (Les Républicains), de ceux qui se rassemblent autour de la défense d'une laïcité et d'une République supposées en péril (Alain Finkielkraut, académicien, essayiste et animateur radio), ou encore d'un universitaire se prononçant sur le sujet (Daniel Stoecklin).

Notre travail ne cherche pas à mettre en évidence les représentations des identités en circonscrivant les traits distinguant le 'nous' du 'eux' car ce serait rester au même niveau des discours que l'on analyse en proposant une retraduction dans le langage universitaire, tout en réifiant ces traits représentatifs ${ }^{\mathrm{I}}$.

${ }^{r}$ Nous nous distinguons également des travaux en CDA qui mettent au jour une grammaire des stratégies d'assimilation et de différenciation à travers les opérations discursives de construction des identités et des procédés de légitimation (par

Comment citer ce chapitre :

Magaud, V. 20I9. "Les agressions à Cologne au prisme de doxaï et d'enjeux politiques : construction et naturalisation des identités ». In: Françoise Sullet-Nylander, María Bernal, Christophe Premat \& Malin Roitman (eds.). Political Discourses at the Extremes. Expressions of Populism in Romance-Speaking Countries. Stockholm Studies in Romance Languages. Stockholm: Stockholm University Press, pp. 26I-277. DOI: https://doi.org/Io.I6993/bax.m. License: CC-BY 
Notre approche consiste plutôt à poser cette question du 'soi' et de l'autre' en termes de positionnements identitaires, car celle-ci se pose dans des contextes de luttes symboliques et territoriales, ou encore lorsque les identités imaginaires prévalent sur les identités réelles générant des " logiques de discrimination et d'exclusion ou, au contraire, [...] des formes d'admiration ou de glorification » (Lamizet $20 \mathrm{I} 5: 4 \mathrm{I}$ ). Par ailleurs, c'est la nature de la relation à l'Autre qui est vecteur de questionnements et d'affirmations identitaires. L'Autre est par ailleurs souvent un construit qui sert d'exutoire aux fantômes qui nous hantent, ou sert des intérêts qu'ils soient politiciens ou de l'ordre du politique. C'est donc en envisageant la question des identités autour d'un événement sidérant, c'est-à-dire qui surprend par son étrangeté et bouleverse l'idée de soi, que les positionnements vont émerger. La notion de positionnement identitaire fait référence à la fois à l'identité énonciative qui « se maintient à travers l'interdiscours par un travail incessant de reconfiguration » (Charaudeau \& Maingueneau 2002 : 453-454), en fonction des positions des concurrents dans le même champ - il s'agit donc se différencier tout en restant affilié -, et aux visions du monde et des positions qu'on y occupe, que l'on cherche à maintenir ou à changer (Bourdieu \& Fritsch 2000), la référence identitaire y étant prééminente. Parler de positionnement identitaire permet de rendre compte de ce construit dans la relation à soi et aux autres, des enjeux et des fantasmes qui s'y jouent.

Autour de cet événement sidérant que sont les agressions à Cologne, nous avons confronté trois types de discours : le politique, l'universitaire, l'intellectuel. Au-delà de leurs particularités, émergent des dominantes interdiscursives communes qui participent à la construction des identités et trahissent des positionnements identitaires. C'est cet interdiscours que nous interrogerons par une analyse argumentative et sémantique des données linguistico-discursives, les ethè discursifs (Maingueneau2002), les imaginaires qui s'y légitiment à travers les citations, les formules et les exemples historiques. Il s'agit plus largement de voir comment la/le politique surdétermine ces positionnements identitaires sur Soi/Autre.

exemple Reisig, M. \& Wodak, R. (200I) : Discourse and discrimination. Rhetorics of racism and antisemitism, London, Routledge). Notre propos vise à saisir la dynamique des positionnements identitaires via l'appropriation et l'accommodation de discours autres. 


\section{Contexte, corpus et méthodologie}

Après avoir introduit le contexte et les derniers résultats des enquêtes sur les agressions à Cologne, cette partie inaugurale présente le corpus constitué de quatre interventions, relevant des champs politique, intellectuel et universitaire, et la méthodologie utilisée. Cette dernière conjoint analyse du discours française et argumentation rhétorique, celle-ci s'inscrivant dans la lignée des travaux de Perelman et OlbrechtTyteca ([I958]I992) et, à leur suite, Plantin (I996) et Amossy (2000).

\subsection{Rappel des faits}

Des agressions à l'encontre de femmes sont commises le soir du nouvel an 20I6, en Rhénanie, à Cologne ; d'autres agressions similaires, dans une proportion moindre, sont rapportées dans d'autres villes allemandes et d'Europe du nord. Ulrich Bremer, procureur de la ville de Cologne, fait alors état de I088 plaintes pour agressions sexuelles et vols avec coups et blessures. Les personnes inculpées, 73 au total, sont d'après Bremer des demandeurs d'asile, des personnes arrivées illégalement, et comptent majoritairement des nord-africains. Jeune Afrique rapporte le 22 janvier l'existence d'un réseau de délinquants marocains ayant fait l'objet d'investigations de 20I4 à fin 20I 5 par la police de Düsseldorf dans le cadre du projet Casablanca visant à enquêter sur une délinquance nord-africaine en forte expansion. Ces réseaux auraient pu sévir à Cologne, la technique pour approcher les victimes étant la même que celle recensée par la police de Düsseldorf. Des enquêtes sont toujours en cours mais des inculpés ont été relaxés².

Sans certitude quant aux agresseurs, les réactions se sont vite fait entendre pour dénoncer, d'une part, le manque de réactivité des autorités et des médias et, d'autre part, la politique d'accueil de réfugiés en Europe initiée par Angela Merkel, dans un contexte de violences mondiales, avec pour corollaire un afflux de réfugiés. En France, les explications hâtives de ces agressions n'ont pas tardé à paraître, à commencer par celles de l'écrivain et journaliste algérien Kamel Daoud (Le Monde, 3 I janvier ; Sunday Review, I2 février2or6), qui fustige le monde musulman par les tabous qui pèseraient sur la sexualité dans

${ }^{2}$ https://www.legrandsoir.info/le-tribunal-de-hambourg-relaxe-les-accuses-de-lanuit-du-nouvel-an-a-cologne.html. The Local du I4.02.20I7 rapporte que les agressions à Frankfort ont été inventées : https://www.thelocal.de/20170214/ mass-sexual-assaults-by-refugees-in-frankfurt-completely-made-up. 
certains pays arabes. En réponse à ces tribunes, un collectif composé d'universitaires et de journalistes réagit aux propos de Daoud dans la rubrique Idées $\mathrm{Du}$ Monde le I I février lui reprochant sa vision clivée, psychologisante et essentialiste de deux mondes au détriment de considérations politiques et sociales. ${ }^{3}$

Nous ne reviendrons pas sur cette polémique. Nous nous sommes plutôt intéressées aux réactions à chaud à portée identitaire émanant du monde politique et intellectuel de notre côté de la Méditerranée afin de voir comment se construisent les identités dans un contexte de tensions politiques et idéologiques ainsi que, pour reprendre Bourdieu (2000), les principes de vision et de division qui cherchent à s'imposer.

\subsection{Corpus}

Notre étude s'appuie sur les interventions de personnalités dans les médias numériques, à support papier ou radiophonique ou encore dans des organes politiques comme le Sénat. L'objectif étant de saisir les lieux où se joue la redéfinition de discours tenus ailleurs et par d'autres, elle privilégie le parcours d'unités non topiques dans une perspective analytique c'est-à-dire la circulation d'éléments linguistiques ou discursifs non rattachés à un genre de discours particulier ${ }^{4}$ et d'empan large. Elle porte, d'une part, sur un article de Marine Le Pen, chef de file du parti Front National (extrême-droite), qui réagit aux événements dans la rubrique Tribune libre du journal en ligne L'Opinion's le I 3 janvier sous le titre "Un référendum pour sortir de la crise migratoire ». Elle s'appuie, d'autre part, sur l'allocution de Valérie Boyer, députée Les Républicains (droite) des Bouches-du-Rhône, membre de la commission aux affaires sociales, qui s'adresse le I 5 janvier au premier ministre Valls au Sénat au sujet des agressions ${ }^{6}$. Par ailleurs, elle porte sur les interventions d'Alain Finkielkraut, philosophe, essayiste, journaliste et

3 http://www.lemonde.fr/idees/article/20 I 6/02/I I/les-fantasmes-de-kamel-daoud_ 4863096_3232.html\#gSX8GObsdc2qrb3d.99

4 Voir Maingueneau, P. (2005)." L'analyse du discours et ses frontières". Marges linguistiques 9, et le découpage des "objets" de l'analyse de discours française qu'il propose en unités topiques et unités non topiques qui correspondent respectivement à une démarche intégrative et une aproche analytique

5 http://www.lopinion.fr/edition/politique/marine-pen-referendum-sortir-crisemigratoire-94568. L'Opinion est un journal pro-européen, probusiness et à tendance libérale dont le président et directeur de rédaction, N. Beytout est l'ancien président des Echos (finances) et ancien directeur de rédaction du Figaro (quotidien de droite) ${ }^{6}$ https://www.youtube.com/watch?v=In7UQ5nyO_c 
académicien, qui anime l'émission Répliques sur France Culture depuis I985, et auteur d'essais, qui lui ont valu d'être qualifié de néoréac ${ }^{7}$ et de décliniste. Il intervient sur les agressions dans l'émission l'Esprit de l'escalier sur RadioJuive Communautaire ${ }^{8}$ qu'il anime en tandem avec Élisabeth Levy (journaliste et directrice de rédaction dans la revue en ligne Causeur, classée à droite), le so janvier et le 26 à l'École polytechnique retransmis par Le Figaro.tv ${ }^{9}$, quotidien classé à droite. Daniel Stoecklin enfin, professeur à l'Institut Universitaire Kurt Bosch et à l'Institut International des Droits de l'Enfant à Sion en Suisse, spécialiste de l'enfance et des droits de l'enfant, intervient sur les agressions dans Jet d'encre ${ }^{\text {I0 }}$ le 26 janvier 2016 sous le titre "Une agressivité civilisée ». Cette revue, rassemblant essentiellement des universitaires, présente les caractéristiques d'un quotidien avec les rubriques usuelles (société, culture, etc.) et se veut le lieu d'une délibération citoyenne privilégiant l'analyse et la pluralité des points de vue, à l'image de The Conversation.

\subsection{Méthodologie et axes d'étude}

En focalisant notre attention sur l'interdiscours, entendu comme "l'ensemble des unités discursives (relevant de discours antérieurs du même genre, de discours contemporains d'autres genres) avec lesquelles un discours entre en relation implicite ou explicite " (Charaudeau $\&$ Maingueneau 2002 : 324), et les conditions sociopolitiques dans lesquelles ces phénomènes sont produits, notre analyse vise à faire émerger ces fictions que l'on entretient sur soi et sur l'autre, surdéterminées par les tensions et les enjeux qui structurent les différents champs concernés. L'analyse du discours considère les discours comme poreux les uns aux autres et les phénomènes de reprises montrent une impossible isomorphie entre un discours et un groupe défini, l'identité se constituant dans les processus de redéfinition, de répétition, de travestissement de « discours produits dans d'autres discours, antérieurement à lui et indépendamment de lui » (Maingueneau I99I : I6I). Les phénomènes interdiscursifs sont corrélés aux schèmes argumentatifs sous-jacents ou

\footnotetext{
7 Lindenberg D. (2002), Le rappel à l'ordre : enquête sur les nouveaux réactionnaires, Paris, Le Seuil

${ }^{8}$ https://www.youtube.com/watch?v=-jpx4jGaO $5 \mathrm{I}$ https://www.youtube.com/watch? $\mathrm{v}=-\mathrm{jp} \mathrm{x}_{4} \mathrm{j} \mathrm{GaO}_{5} \mathrm{I}$

9 http://www.lefigaro.fr/vox/culture/20 I 6/0I/28/3 I006-20I 60 I 28ARTFIGo0230grandes-rencontres-du-figaro-alain-finkielkraut-immortel.php

to http://www.jetdencre.ch/une-agressivite-civilisee-9753
} 
topoï faisant émerger l'arrière-plan doxal et idéologique qui sous-tend les discours et qui nous renseigne sur ce que l'on fantasme sur soi et sur l'autre. L'argumentation y est envisagée dans sa dimension dynamique, dialogique où les lieux argumentatifs, les topiques et les ethè y jouent un rôle essentiel. Les identités sont donc appréhendées à travers les analogies, les topiques événementielles qui structurent les formulations, les nouveaux référents qui s’imposent, ces phénomènes conférant aux fragments de discours réappropriés un caractère naturel.

Aussi la notion de positionnement rend-elle compte à la fois de la façon dont les identités se délimitent et se déterminent dans l'interdiscours au moyen d'opérations discursives et argumentatives, dans une situation de confrontation et de concurrence. Le positionnement identitaire est appréhendé dans la présente étude, d'une part, au travers des citations qui, en plus de conférer une légitimité au citant par contamination avec l'auteur de la citation, sont accommodées au sens commun et au contexte. Cette transmutation en sens commun d'un système de pensée qui lui est antérieur ou doxopraxie procède de normes qui s'actualisent par des topoï (Sarfati 2002). Elle transforme par ailleurs les propos premiers en médiations partisanes car la citation est arrimée au dogme idéologique du citant. Le positionnement identitaire procède alors d'endoxons ${ }^{\mathrm{II}}$ qui émergent du raisonnement dans lequel la citation est prise.

Le positionnement identitaire procède, d'autre part, de l'usage d'exemples historiques qui permettent d'établir une analogie entre un événement antérieur (phore) et un événement présent (thème), et expliquer ce dernier à la lumière des leçons tirées du premier supposé semblable (Aristote I99I). Ce rapprochement vise à faire émerger une propriété commune entre phore et thème (Perelman I992) et à faire admettre une thèse, le positionnement identitaire transparaissant via ce trait commun que prend le comparant au contact du comparé et justifiant les attitudes de rejet ou/et de glorification.

Enfin, le positionnement est appréhendé à travers la circulation de formules, c'est-à-dire " un ensemble de formulations qui, du fait de leurs emplois à un moment donné et dans un espace public donné, cristallisent des enjeux politiques et sociaux que ces expressions contribuent dans le même temps à construire » (Krieg-Planque 2009 : 7). Elles permettent de fait de se singulariser et de délimiter une identité

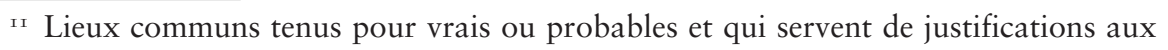
propos avancés (voir Plantin I996, 2002) 
grâce aux référents que les personnes leur accolent et qui jouent en faveur d'une différenciation polémique.

\section{Phénomènes interdiscursifs, construction et naturalisation des identités}

La notion de positionnement permet de rendre compte de la construction de ces fictions clivantes autour du 'nous' et du 'eux' et également du jeu médiatique et politique qui consiste à entretenir une polarisation entre différents groupes.

Pour rendre compte des positionnements identitaires dans un climat de tensions et conflits politiques à la fois internes et externes et de leurs différents rôles, notre focale se centre sur les trois phénomènes discursifs sus-cités. La citation tout d'abord joue en faveur d'un modèle dont on se réclame et d'un anti-modèle qu'on fustige, légitime une frontière entre le 'nous' et le 'eux' et renforce une idéologie au moyen de ces médiations "partisanes ", celle d'une hégémonie à retrouver comme vecteurs d'action. Les formules ${ }^{12}$ ensuite qui, à travers le fonctionnement de terrorisme sexuel, de civilisation française notamment, réactivent un imaginaire sur l'autre. Elles s'inscrivent sur fond de polémique et fonctionnent comme totem en conférant une identité démarcatrice dans le champ ou inter-champ où elles opèrent. L'exemple historique, enfin, celui de la place Tahrir, nous éclaire sur l'émergence d'une nouvelle mission émancipatrice de l'occident.

\subsection{Le rôle des citations et doxopraxie}

La candidate $\mathrm{M}$. Le Pen accommode l'événement au prisme idéologique de son parti (l'immigration doit être réduite, les agressions apportant une justification supplémentaire à ce leitmotiv) et y associe un argument d'autorité d'autant plus efficace qu'il concerne une figure du féminisme, Simone De Beauvoir. La citation mobilisée, se situant dans le contexte émancipateur des années 60-70 en France, est décontextualisée et déshistoricisée en lui associant les faits présents. La menace prophétisée par De Beauvoir faisant référence au travail d'éternisation des structures de la division sexuelle, pour reprendre Bourdieu (I998), ,33

\footnotetext{
${ }^{12}$ Il s'agit de collocations constituées de lexies suivies d'une expansion adjectivale (relationnel) et qui, par les changements sémantiques qui s'opèrent, présentent des enjeux idéologiques et de positionnement dans un champ

I3 Bourdieu, P. (I998). La domination masculine. Paris: Editions du seuil
} 
et les valeurs axiologiques qui lui sont attachées, est instanciée (crise migratoire), associée à des arguments-objets (atteinte à l'intégrité physique, contrôle social, réduction des libertés et asservissement) et repolitisée (stop à l'immigration) en y associant des actants-opposants, les migrants, et en l'assujettissant au dogme idéologique :

(I) Le droit à l'intégrité corporelle, de quelque sexe que l'on soit, est un droit parmi les plus essentiels. Ce droit est aujourd'hui attaqué pour nombre de femmes. Que la barbarie puisse s'exercer de nouveau à l'encontre des femmes, du fait d'une politique migratoire insensée, me remplit d'effroi. Je repense à ces paroles de Simone de Beauvoir: "N'oubliez jamais qu'il suffira d'une crise politique, économique ou religieuse pour que les droits des femmes soient remis en question ", et j'ai peur que la crise migratoire signe le début de la fin des droits des femmes. Atteinte à l'intégrité physique, contrôle social, réduction des libertés et asservissement: on sait que la pente est glissante. Sur ce sujet comme sur les autres, les conséquences de la crise migratoire étaient pourtant prévisibles ${ }^{\mathrm{T}}$.

Il ne s'agit donc plus de dénoncer la rémanence des structures patriarcales en France mais de montrer que cette menace pesant sur l'endogroupe vient de l'extérieur, les migrants étant de fait assimilés aux agresseurs passés et jouant le rôle de repoussoir afin de faire avaliser la thèse du rejet de l'immigration. Le topos des inséparables sur lequel repose le raisonnement (la crise migratoire implique nécessairement une remise en question des droits des femmes) naturalise et érige en règle le lien entre présence des migrants et fin des droits des femmes. La candidate joue également sur la proximité analogique en laissant entendre, au moyen de présupposés et d'inchoatifs, que de pareilles agressions aient été monnaie courante ou qu'elles menacent les droits des femmes ( « que la barbarie puisse de nouveau s'exercer à l'encontre des femmes "; " les femmes ne peuvent plus jouir comme l'homme de ces mêmes droits "; "la crise migratoire signe le début de la fin des droits des femmes "; " libertés très chères acquises de haute lutte "; "nouvelle forme de régression "). Les migrants sont donc bel et bien assimilés aux agresseurs passés et cette analogie permet à la candidate de condamner l'immigration, fer de lance de son parti, mais aussi de jouer sur la peur d'un retour en arrière d'une société pétrie de discours progressistes.

${ }^{14}$ Voir note 8. 
A. Finkielkraut réfute au moyen de négations polémiques les explications en termes de dominants-dominés et celles qui pointent du doigt le rejet des musulmans en occident. La convocation de propos de Fethi Benslama, psychanalyste tunisien directeur de l'UFR de l'Université Paris Diderot, apporte une triple légitimité au discours de Finkielkraut. Il permet de neutraliser les oppositions en convoquant une figure d'autorité, caution renforcée par le fait que Benslama est lui-même issu d'un pays musulman. Les agressions à Cologne sont assimilées à l'idée que les femmes sont opprimées par l'islam, idée que la citation vient corroborer :

(2) Fethi Benslama ajoute dans sa Déclaration d'insoumission à l'usage des musulmans et de ceux qui ne le sont pas que l'oppression des femmes ne dégrade pas seulement la femme mais organise dans l'ensemble de la société l'inégalité la haine de l'altérité la violence par le pouvoir mâle il faut aider les musulmans à se défaire de cette oppression sinon voilà voilà ce qui arrive ${ }^{15}$.

La citation conduit à la conclusion suivante, reposant sur le lieu des inséparables : si on veut enrayer de pareilles agressions, alors il faut se défaire de l'islam; et l'occident est investi de cette mission. Les migrants sont donc définis par le seul prisme de la religion ${ }^{16}$ et la haine de la femme que l'islam instillerait. Il s'agit en fait de réactualiser le discours universaliste en prenant comme valeur d'émancipation la libération des femmes, d'ériger l'endogroupe comme le modèle à suivre, exempt des traits attribués aux sociétés arabo-musulmanes, et qui en constitue l'envers : égalité, altérité, non patriarcal. L'identité de l'exogroupe émerge de fait selon le lieu des contraires : si l'oppression des femmes est imputable à l'islam, alors leur libération procède d'une négation de l'islam. L'endogroupe est investi de ce pouvoir de libération. On ôte toute épaisseur politique à l'autre, réduit à une altérité radicale et à sa seule appartenance confessionnelle qui plus est répressive ; les cultures sont hiérarchisées, l'occident apparaissant bienfaiteur et libérateur.

Se réaffirme, à travers ces médiations "partisanes ", la supériorité de l'occident, les droits des femmes devenant le fer de lance d'une hégémonie à reconquérir face à un dark age dans lequel l'islam risque de plonger l'humanité.

${ }^{15}$ A. Finkielkraut, l'Esprit d'escalier à Radio communautaire juive le ıo janvier 2016 (voir note II). A noter que les extraits oraux font l'objet d'une transcription orthographique non ponctuée

I6 En oubliant de considérer et les structures du pouvoir de ces sociétés, en réalité sécularisées, et leur contestation à travers l'émergence de mouvements de radicalisation. 


\subsection{Les formules, leur remotivation et leurs enjeux identitaires}

Dans le discours politique, les formules lors de leur parcours discursif perdent ou gagnent des traits distinctifs en fonction des enjeux et des contextes de luttes symboliques et politiques. A ce titre, la circulation du lexème civilisation est éclairante car elle montre comment l'intertexte est accommodé au contexte et aux enjeux de pouvoir et sert de dissociation catégorielle polémique, jouant comme phénomène de différenciation.

Le sens interdiscursif de civilisation en emploi absolu s'est confondu, au I 8ème siècle, avec civilisation universelle que doit atteindre toute humanité, c'est-à-dire le progrès et le développement scientifique ; il se confond aujourd'hui avec celui de culture. Civilisation est plus communément associé à un relationnel et évoque un ensemble de sociétés avec des configurations historiques particulières présentant des traits communs dans le temps et l'espace (Obadia 20I6). Dans le discours politique, le terme est reconvoqué avec l'usage idéologique emblématique que Nicolas Sarkozy en a fait dans son discours de Dakar en 2007. Chez M. Le Pen, civilisation française est remotivée et prend alors par contamination avec le co-texte les traits de 'sécurité de tous' et 'les droits des femmes': « [...] A toutes les raisons qui commandent la réduction drastique de l'immigration, s'ajoutent désormais des impératifs qui touchent aux fondements même de la civilisation française: la sécurité de tous, et les droits des femmes[...] ». ${ }^{17}$

Civilisation française ainsi assimilée à l'état régalien, conformément à la perspective politique frontiste, permet de conférer à la France le monopole des droits des femmes. Cet emploi perfectif (on lui accole des traits et une localisation), son indexation sur des événements contingents rapportés à la France repose sur le lieu de l'existant ${ }^{18}$ et permet à la candidate de se positionner du côté de la vérité des faits de façon à faire avaliser sa thèse sur l'immigration et la souveraineté nationale. Cette restriction du sens interdiscursif à deux référents vise à se démarquer d'une part des partis pro-européens et à faire adopter pour seule légitimité l'idée d'un État régalien. Elle légitime un imaginaire où celui-ci s'est imposé depuis toujours comme l'atteste l'utilisation du mot civilisation qui donne un ancrage historique aux référents tout en réaffirmant implicitement que la France a le monopole des droits de

\footnotetext{
${ }^{17}$ Voir note ${ }^{8}$

I8 «Les lieux de l'existant affirment la supériorité de ce qui existe, de ce qui est actuel, de ce qui est réel, sur le possible, l'éventuel ou l'impossible. »(Perelman, op. cit.: I26)
} 
l'Homme. En effet, la formule et l'imposition des référents sociaux qui lui sont associés impliquent que les droits des femmes sont une spécificité française et que leur absence et celle d'État régalien renvoient à un État non civilisé. L'emploi du mot civilisation n'est pas innocent car les traits de progrès et de rayonnement y sont capitalisés en langue. Il s'agit par ailleurs via cette formule de se distinguer de la droite et de leur formule totem "l'identité française » et de réaffirmer la souveraineté nationale par le lieu de l'ordre qu'impose la visée argumentative de la formule. Ce médiateur symbolique permet de jouer sur la fibre nationale en flattant l'ethos collectif et d'attribuer aux étrangers une image inversée, le lieu de l'insécurité, du non-droit, de la régression.

La formule terrorisme sexuel apparaît en $20{ }_{3} 3$ quand l'Express du 6 mars rapporte les propos d'Inas Mekkawy du mouvement de défense des droits des femmes Baheya ya Masr: "Nous voulons que le terme +harcèlement+ ne soit plus utilisé. Ce dont il s'agit aujourd'bui, c'est de terrorisme sexuel ${ }^{19}{ }^{\prime}$. Cette déclaration fait suite aux nombreuses agressions à caractère sexuel dont les femmes égyptiennes ont fait l'objet lors de la révolte populaire pour renverser le régime de Hosni Moubarak et ultérieurement lors de manifestations de célébration de ce soulèvement.

La formule, reprise dans la presse avec des guillemets en faisant référence à ces mêmes agressions, figure par la suite en usage, ne renvoyant plus seulement à ces événements historiquement situés. Dans le discours de Valérie Boyer, elle se rapporte aux agressions à Cologne, devient un moyen de disqualifier le gouvernement et de fustiger l'irresponsabilité de ce dernier face aux migrants. Terrorisme renvoie à l'idée qu'un groupe refuse la relation étatique, nie ce monopole de la violence qui échoit à l'État. La formule assimile les agressions sexuelles à une délégitimation de l'État en s'attaquant aux femmes. Dans la situation égyptienne, le syntagme renvoie à l'idée que des groupes à l'intérieur de la nation s'opposent et s'imposent les uns aux autres dans une lutte pour le pouvoir et, dans les propos de Mekkawy, que les femmes deviennent un enjeu politique pour délégitimer le mouvement ou certains groupes. Les femmes agressées considèrent que ces actes visent "à les exclure de la vie publique et à les punir de leur participation au militantisme politique et aux manifestations. Elles sont aussi une tentative de ternir

i9 https://www.lexpress.fr/actualites/r/monde/en-egypte-des-femmes-en-guerre-contrele-terrorisme-sexuel_I227354.html 
l'image de la place Tahrir et des manifestants en général ${ }^{20}{ }^{\circ}$. Le terrorisme sexuel serait ainsi un moyen de miner le soulèvement de l'intérieur en s'attaquant aux femmes tout autant qu'une contestation de la légitimité politique des femmes. Elle présente les traits +violence +délégitimation politique +délégitimation d'un groupe sexuel +intergroupal.

Dans le discours de Valérie Boyer, qui procède par questions rhétoriques afin d'élargir son point de vue à celui de l'ensemble des Français, et de se présenter comme leur porte-parole, la formule dans son parcours perd le trait de délégitimation politique à l'encontre de groupes d'opposition ou d'un groupe sexuel et prend ceux de +chasse à l'homme +guerre :

(3) À la lumière de ces événements ${ }^{21}$, comment ne pas être choqué par le manque de réactivité des autorités européennes ? Où sont les féministes ? Sommes-nous en train d'assister à une nouvelle forme de terrorisme, un terrorisme sexuel où l'on considère les femmes comme du gibier les français sont inquiets ${ }^{22}$.

Ainsi les migrants sont présentés comme refusant le contrat qui lie les citoyens et l'État et comme force brute voire bestiale ; la figure du migrant fait ici écho à l'imaginaire rémanent autour du barbare qui s'impose par la force et en s'accaparant les femmes du pays à conquérir. Inversement, le discours se structure en contrepoint sur un imaginaire irénique autour de la situation des femmes en Europe versus un imaginaire dysphorique autour du hors culture qui caractériserait les migrants.

Si la formule permet de jouer sur les imaginaires et les peurs qu'elle suscite, elle constitue également une formule-totem clivante dans la mesure où elle représente une ligne de fracture entre les pro-migrants et les anti-migrants, donne une identité à la politique et à la vision de la candidate. Il s'agit de se distinguer d'une gauche qualifiée implicitement d'irresponsable qui n'anticipe pas les dangers ou les problèmes et refuse d'appeler un chat un chat, et de s'aligner sur les discours d'extrême-droite faisant de l'immigration une menace.

Dans le champ universitaire, la contribution de Stoecklin dans la revue Jet d'encre au lendemain des événements vise à tirer un enseignement des attentats commis à Paris susceptibles de s'appliquer aux événements de Cologne : « [...] on peut commencer à entrevoir que le point commun entre ces deux phénomènes, est que les agressions

\footnotetext{
${ }^{20} I d$.

${ }^{21} \mathrm{~V}$. Boyer fait bien sûr référence aux agressions à Cologne

${ }_{22}$ Voir note $^{6}$
} 
sexuelles tout comme les attentats terroristes sont des comportements extrêmement désorientés $[\ldots] »^{23}$.

L'auteur, à la lumière des travaux de N. Elias sur la société curiale ${ }^{24}$, oppose les sociétés où prévaut l'autocontrôle grâce à la civilisation des mœurs et celles où prévaut l'exocontrôle, dicté de l'extérieur. La " civilisation des mœurs ", concernant surtout l'occident, et les sociétés qui en sont dépourvues se déclinent respectivement à travers des traits opposés: distanciation et autoréflexivité $v s$ non distanciation par rapport au dogme, agressivité civilisée dont l'humour et la fête sont l'expression vs agressivité incontrôlée et généralisée dont la disparition de la fête est l'expression, régulation de l'agressivité par l'État $v s$ monopole de la violence à la place de l'État :

(4) En quelque sorte, en suivant Elias, on peut voir l'humour et l'ironie comme de l'agressivité civilisée. [...]De Socrate à Montaigne (" connais-toi toi-même »), l'Occident a valorisé la distanciation et l'autoréflexivité. Le foisonnement de la littérature en est l'indice le plus évident. Là où n'existe qu'un seul livre (le livre) il n'y a pratiquement pas d'autoréflexivité possible, et donc pas d'autonomie. [...] une civilité aujourd'hui relativement mondialisée, reposant sur l'auto-contrôle, la distanciation par rapport à l'autorité et à soi-même (réflexivité) et n'autorisant l'agressivité que sous la forme euphémique de l'humour et de l'ironie. Ce que ne supportent pas les fondamentalistes, c'est "la civilité des autres " (dont la nôtre) et l'agressivité régulée qu'elle suppose.

[...] Face aux agressions de Cologne, nous devons poser les bonnes questions, et ne pas nous laisser enfermer par la stigmatisation ou la récupération politique. Les points communs doivent stimuler une réflexion plus " haute " : dans tous les cas, on constate que faire la fête est possible quand l'agressivité est régulée, et que cela disparaît quand l'agressivité est généralisée ${ }^{25}$.

Cette catégorisation binaire et inversée de type culturaliste réactive et légitime un imaginaire sur les sociétés arabo-musulmanes apparaissant comme des sociétés anhistoriques qui en sont à une étape ${ }^{26}$ de leur progression vers

${ }_{23}$ Voir note ${ }^{\mathrm{I}}$

${ }^{24}$ On peut s'interroger d'une part sur la mobilisation d'une analyse de situations historiquement et géographiquement situées (l'Europe au temps de la royauté) transformée en heuristique pour parler de situations contemporaines et d'espaces géographiques différents, sans qu'ils constituent directement le terrain du chercheur.

${ }_{25}$ Voir note $^{9}$

26 « [...] si on suit la thèse d'Elias, on devrait conclure que les fondamentalistes vivent encore dans une 'dynamique' dominée par l'impulsivité (que la 'dynamique de l'Occident' a largement résorbé) ». 
la « civilisation des mœurs ", caractérisée par le contrôle des émotions, par des expressions et codes différenciés et par la délégation du pouvoir et de la violence à l'État. Cet imaginaire est imprégné d'une conception évolutionniste des cultures mais aussi d'une vision toute hégélienne où historicité rime avec État. Il s'inscrit dans un modèle hiérarchique des cultures où l'occident représente le modèle à suivre. Par ailleurs, l'auteur qualifie la violence liée aux attentats et agressions de " conflictualité normative » qu'il oppose à « choc des civilisations ", formule éponyme du livre de Samuel P. Huntington, qui a été reprise à l'envi après les attentats de Paris ${ }^{27}$. Il s'agit pour l'auteur de se positionner et dans le champ universitaire et dans le champ médiatique. En effet, bien que l'auteur s'en défende, il procède de la même façon que Huntington (I997) en réifiant des traits censément représentatifs de deux mondes, les " agresseurs " présentant de fait un handicap lié à leur identité monolithe puisque seul l'occident connaitrait des expressions plurivoques et serait donc plus apte à gérer les différences ${ }^{28}$ :

(5) Il faut commencer par comprendre que leur violence exercée sur des victimes expiatoires, est une réponse sans doute inconsciente à leur propre désarroi face à une conflictualité normative par rapport à laquelle ils n'arrivent pas à prendre personnellement position et éprouvent donc le besoin de se ranger dans un camp qui leur offre la vision rassurante d'une conception manichéenne du monde (les bons et les mauvais). C'est ici que l'on voit pourquoi la «conflictualité normative » est une notion plus adéquate que l'expression " choc des civilisations " : elle ne réifie pas les " civilisations ", mais fait au contraire entrevoir des processus de subjectivation différents, des processus de construction identitaires différentiés ${ }^{29}$.

Il s'agit donc avec cette formule de se démarquer des médias classiques, qui ont et continuent pour certains d'accueillir favorablement la conception huntingtonienne des relations internationales, mais également de se positionner dans le champ universitaire en imposant une idée-force qui permette de se définir par rapport aux concurrents.

${ }_{27}$ Par exemple, Jean-Louis Bourlanges, ancien député européen et professeur associé à Sciences Politiques à Paris ( «Les violences à Cologne nous font découvrir le "choc des civilisations” au quotidien», Le Figaro, I6.or.20I6) ; A. Finkielkraut parle de " choc des cultures".

28 «Contrairement à l'Occident, qui a favorisé l'intériorisation des normes, et tolère donc leur interprétation personnelle, les régions du monde dominées par une pensée religieuse monothéiste considèrent comme 'sacrée' une forme précise de 'civilité', qui impose des pratiques incontournables et non-interprétables (dont le port du voile pour les femmes, le mariage précoce, l'excision et la circoncision) ».

29 Voir note ${ }^{13}$ 


\subsection{Le précédent historique : matrice narrative accommodée à l'étalon 'émancipation'}

Finkielkraut utilise, dans les discours recueillis, le précédent de la place Tahrir. Le fait historique comparant est décontextualisé et est opérée une lecture sélective, anhistorique et simplificatrice pour pouvoir être accommodée à la situation présente. Ainsi place Tahrir devient le symptôme de l'échec des révolutions arabes infiltrées par l'islamisme:

(6)[...] ce qui s'est passé à Cologne rappelle ce qui s'est passé à place Tahrir pendant le printemps arabe des hommes agressant sexuellement des femmes pour les chasser de l'espace public on a beaucoup parlé des printemps arabes on est tous très malheureux de l'évolution de l'hiver qui a suivi mais quand on sait ce qui s'est passé place Tahrir on peut dire que l'hiver était dans le fruit c'était là ${ }^{30}$

Un sens événementiel est capitalisé dans la formulation synecdotique qui renvoie à la révolution égyptienne de $20 \mathrm{II}$. On peut ainsi dérouler une topique événementielle qui structure le syntagme autour d'actants, d'un lieu (place Tahrir), de dates (du 25 janvier jusqu'à la chute de Moubarak), de moyens, d'actions et d'un but. Cette matrice narrative est cependant simplifiée par la topique qui émerge de son usage comme on peut le voir dans le tableau suivant :

Tableau : Lecture analogique des agressions à Cologne. Copyright : Véronique Magaud. Licence : CC BY

\begin{tabular}{lll}
\hline & $\begin{array}{l}\text { L'événement sous sa forme } \\
\text { synecdotique }\end{array}$ & Lecture analogique \\
\hline Lieu & Place Tahrir & Tahrir = Cologne \\
Actants & $\begin{array}{l}\text { Mouvement de la jeunesse du 6 } \\
\text { avril et des milliers d'Égyptiens/ } \\
\text { le pouvoir et ses partisans } \\
\end{array}$ & $\begin{array}{l}\text { Des hommes vs des } \\
\text { femmes }\end{array}$ \\
& (Moubarak et les pro-Moubarak) & \\
Moyens et & Occupation et services jusqu'à être & Agressions sexuelles \\
But & une ville dans la ville & \\
& Occupation en vue de la chute du & Éviction des femmes de \\
& gouvernement & l'espace public \\
\hline
\end{tabular}

$3^{\circ}$ Voir note ${ }^{12}$ 
Cette lecture 'genrée' évacue toute la portée politique du soulèvement au profit d'une violence à l'encontre des femmes ${ }^{3}$. Les incidents ${ }^{32}$ qui ont émaillé l'occupation sont érigés en représentation optimale de façon à maximiser la continuité entre ces événements et les événements qui ont eu lieu à Cologne en vertu du lieu du tout et de la partie. Place Tabrir devient ainsi l'emblème de l'oppression des femmes qui plus est par l'islam comme Cologne est frappée par le même phénomène. L'association de deux lieux géographiques et de contextes différents permet d'interpréter les agressions à Cologne comme une menace plus large, le fait comparant prenant donc le trait 'infiltrés par l'islamisme'. Ainsi s'impose un récit intermédiaire qui réitère l'idée d'émancipation universelle avec comme étendard, comme valeur émancipatrice, la lutte contre l'islam.

\section{Conclusion}

Notre contribution a montré que les identités qui se construisent dans les discours sont indissociables d'une part des champs dans lesquels les événements prennent place et qui favorisent le jeu concurrentiel, d'autre part des positionnements idéologiques et politiques des intervenants. Les lieux discursifs qui matérialisent des fictions autour des identités révèlent en effet les enjeux de ces catégorisations de l'autre et de soi-même, tout en naturalisant l'imaginaire collectif autour de ces identités. Ils cristallisent les fantasmes entretenus sur l'endogroupe et l'exogroupe. Les migrants endossent ainsi le rôle de miroir repoussant d'une société qui se vit et s'affiche comme progressiste, celui de 'barbares', et sont encore jugés à l'aune d'un évolutionnisme dont nous représenterions l'étape ultime, tandis que le parangon de l'émancipation continue de hanter notre relation à l'orient.

\section{Bibliographie}

Amossy, R. (2000). L'argumentation dans la langue. Paris: Nathan Université. Aristote (I99I). Rhétorique, Paris: Livre de Poche.

Bourdieu, P. (1998). La domination masculine. Paris: Editions du seuil.

${ }^{31}$ Ce que Finkielkraut résume par la métaphore suivante : l'hiver était dans le fruit.

${ }^{32}$ Il ne s'agit pas de minimiser les agressions mais de contester leur association unilatérale à la main mise des islamistes sur le mouvement. Comme dans tout soulèvement, les moyens mis en œuvre pour discréditer sa légitimité et pour le récupérer relèvent de diverses forces en opposition. 
Bourdieu, P. \& Fritsch, P. (2000). Propos sur le champ politique. Lyon: Presses Universitaires de Lyon.

Charaudeau, P. \& Maingueneau, D. (eds.) (2002). Dictionnaire d'analyse du discours. Paris: Seuil.

Daoud, K. (2016). « Cologne, lieu de fantasmes ». Le Monde 3 I janvier. http:// www.lemonde.fr/idees/article/20 I 6/o I/3 I/cologne-lieu-de-fantasmes_ $4856694-3232 . h t m l$

Daoud, K. (2016). " La misère sexuelle dans le monde arabe ». Sunday Review I 2 février. https:/www.nytimes.com/2016/02/I4/opinion/sunday/la-miseresexuelle-du-monde-arabe.html

Hauteville, J.-M., (2016). "Allemagne: y-a-t-il une mafia marocaine à Cologne ». Jeune Afrique 22 janvier. http://www.jeuneafrique.com/296075/ politique/allemagne-y-a-til-une-mafia-marocaine-a-cologne/

Huntington, S. (I997) [I996]: Le choc des civilisations. Paris: éditions Odile Jacob.

Krieg-Planque, A. (2009). La notion de "formule" en analyse du discours. Cadre théorique et méthodologique. Besançon: Presses Universitaires de Franche-Comté.

Lamizet, B. (2015). "Rhétorique de l'identité et discours identitaires ", in A. Richard \& alii (eds), Le discours politique identitaire dans les médias. Paris: L'Harmattan, 25-48.

L'Express (AFP) (2013). "En Egypte, des femmes en guerre contre le terrorisme sexuel », 6 mars.

Maingueneau, D. (I99I). L'analyse du discours. Paris: Hachette supérieur.

Maingueneau, D. (2002). "Problèmes d'ethos». Pratiques I I 3-I I 4, 5 5-67.

Maingueneau, D. (20I4). Discours et analyse du discours. Paris: Armand Colin.

Obadia, L. (2016). "Civilisation ", in O. Christin (ed), Dictionnaire des concepts nomades en sciences humaines. Paris: Métailié, 24 I-255.

Perelman, C. \& Olbrecht-Tyteca, L. (I992) [I958]: Traité de l'argumentation. Bruxelles: Éditions de Bruxelles.

Plantin, C. (2002). "Topos ", in P. Charaudeau et D. Maingueneau(eds), Dictionnaire d'analyse du discours. Paris: Seuil, 576-580.

Plantin, C. (I996). L'argumentation. Paris: Le Seuil.

Sarfati, G.-E. (2002) "Aspects épistémologiques et conceptuels d'une théorie linguistique de la doxa ", in R. Koren \& R. Amossy (eds), Après Perelman: quelles politiques pour les nouvelles rhétoriques? Paris: L'Harmattan, 57-90. 
